\title{
Die Gefässverbindungen der Placentakreisläufe eineiiger Zwillinge, ihre Entwickelung und ihre Folgen.
}

Von

Friedrich Sehatz.

(Fortsetzung.)

\section{Die Acardii und ihre Verwandten.}

Die folgende Arbeit soll die durch die Placentagefässverbindungen bewirkten gegenseitigen Beeinflussungen eineiiger Zwillinge höchsten Grades, wie sie uns in den Acardiis und analogen Bildungen entgegentreten, mit den in der vorigen Arbeit (II., dieses Archiv, Bd. XXVII, XXIX und XXX) besprochenen solchen Beeinflussungen niederen Grades übersichtlich und in einer zusammenhängenden Reihe verbinden. Sie soll damit die Acardii aus ihrer bisherigen unnatürlich isolirten Stellung befreien.

Es kann dies nicht geschehen, ohne dass zugleich auch die Frage nach der Entstehung der Acardii gelöst wird.

Ich bin mir wohl bewusst, dass letzteres Ziel von mir nur in den Hauptzügen erreicht worden ist. Bei der Buntheit, mit weleher uns die zahlreichen Formen der Acardii entgegentreten, bei der grossen Masse literarischen Materials, das meist unvollständig und ungleichmässig beobachtet und beschrieben ist, und bei der Verschiedenheit der Beurtheilungen und Erklärungen, welche die zahlreichen Formen gefunden haben, war es schon eine sehr böse Arbeit (und sie ist erst nach mannigfachen Irrfahrten gelungen), zunächst nur im Groben Ordnung in die widerspruchsvollen Bilder zu bringen. Doch diese Ordnung ist die Hauptsache und wird die bleibende Grundlage für weitere Bearbeitungen bilden.

Die Materie ist aber, selbst nachdem sie jetzt geordnet ist, immer noch schwierig genug und erfordert rom Leser immer noch 
ein förmliches Studium. Ich habe dies zwar durch Abtheilen des Stoffes in Einzelbearbeitungen möglichst zu erleichtern gesucht. Ich habe aber trotzdem mit Absicht vermieden, viele Nebenfragen zu erörtern. Manche von ihnen können ja doch erst dann ihre Lösung finden, wenn bei einmal gewonnener gleichmässiger Richtung in der Untersuchung noch mehr Material wird gesammelt sein.

Jedenfalls mögen künftige Autoren, welche über dieses Thema schreiben, auch wenn sie (etwa in Dissertationen) nur casuistische Beiträge liefern, sich erst genügend in dasselbe vertiefen und ganz genau und allseitig untersuchen, ehe sie Beobachtungen veröffentlichen, Schlüsse ziehen und Meinungen äussern! Unvollständige oder falsch gedeutete Beobachtungen schaden häufig der späteren Erforschung mehr, als sie ihr nützen können.

\section{Literatur.}

\section{Die älteren Autoren über die Aetiologie der Acardii.}

Die Aufzählung der früher über die Entstehung der Acardii ausgesprochenen Meinungen befindet sich in der Arbeit von Tiedemann, Anatomie der kopflosen Missgeburten, Landshut 1813, S. $99 \mathrm{ff}$.

Dort sind als bis dahin ausgesprochene Meinungen mit dem Namen ihrer Urheber, Vertheidiger und Bekämpfer aufgeführt und widerlegt.

a) Die Meinung, dass die kopflose Missgeburt aus einem ursprünglich monströsen Keime oder Ei entstammt;

b) die vielgehegte Meinung, dass ein $n$ Versehen" der Mutter stattgefunden;

c) die Meinung, dass eine von aussen kommende Gewalt die Missgeburt als Verstümmelnng erzeugt und specicll bei der kopflosen Missgeburt den Kopf und die fehlenden Organe auf gewaltsame Weise zerstört habe.

Tiedemann selbst sieht (S. 102) die kopflosen Missgeburten als Hemmungsbildungen an und macht dafür „die Trägheit des Vegetationsprocesses" verantwortlich, welche aus "einer Trägheit des Zeugungsprocesses" entstehe. Dieser reichte wohl hin, den einen Zwilling vollkommen zur Entwicklung zu bringen, nicht aber noch einen vollkommenen zweiten. Entsprechend der übrig gebliebenen Kraft falle derselbe nur als $1 / 4,1 / 2,3 / 4$ Kind aus. 
Dieser Erklärungsversuch von Tiedemann ist sicher nicht besser als diejenigen waren, welche derselbe vorher zurückgewiesen hat. Einer Widerlegung bedarf er in der Jetztzeit ebenso wenig, wie jene ersten.

Freilich sind auch ganz neuerdings Heinungen ernsthaft ausgesprochen, welche kaum weniger naiv sind.

In der Dissertation von Hirschbruch (Das Problem der herzlosen Missgeburten nebst Beschreibung eines Amorphus acardius) heisst es S. 43 und 44 :

„Bei der Betrachtung der niedrigst entwickelten Amorphi müssen wir unwillkürlich an die angeborenen Geschwülste, die zu den Teratomen gehören, denken. Für diese wird momentan eine Keimverirrung angenommen. führen?

Sollte etwa auch zur Amorphusbildung ein abgesprengter Keim

Nach Drieseh's Ansicht kann man vom Furchmosmaterial 1/4, $1 / 2$ oder $3 / 4$, ja wohl jeden beliebigen Bruchtheil nehmen. Wofern der Rest eine gewisse Grösse (1/4?) nicht überschreitet, liefert er ein Thier, das sich nur durch seine mindere Grösse vom normalen unterscheidet.

Das ist ja allerdings nur eine Meinung von Driesch. Es scheint mir aber gar nicht unmöglich, dass ein Bruchtheil, der unter einer hier nicht näher zu erörternden Grenze bleibt, sich nicht mehr normal entwickeh kann, sondern nur zu einem wirren teratoïden Durcheinander von verschiedenen Geweben wird."

Das eigentliche Resultat der Arbeit von Hirscbbruch aber ist: "Zu einem sicheren Resultate über die Aetiologie des „Gemellus placentoparasiticus" (= Acardius) können wir nie kommen. Es handelt sich hier nur um grössere oder geringere Wahrscheinlichkeit der einzelnen Theorien."

\section{Die älteren Autoren über die Circulationsverhältnisse der Acardii.}

Die Meinungen der früheren Autoren über die Circulationsverhäitnisse in den herzlosen Missgeburten sind kurz folgende:

Poujol (Mémoires pour l'Hist. des scienees et des beaux arts, Prevoux 1706) glaubte, dass die von ihm beschriebene Missgeburt nach Art der Pflanzen ernährt worden sei. Das von ihm allein aufgefundene venöse Gefässsystem sollte mit den Placentagefässen als Wurzeln die Nahrung aufnehmen, und mit den Verzweigungen in der Missgeburt an diese abgeben.

Mery (Mémoires de l'Académie des sciences de Paris 1720) glaubte, dass der Blutkreislauf im Acardius durch den mütterlichen Blutkreislauf unterhalten werde. Ebenso

Le Cat (Philos. Transactions for the year 1767, Vol. 57). Er fand in den Acardiis zugleich einen Beweis dafür, dass zwischen Fötus und Mutter Gefässverbindung stattfände. 
Winslow (Mémoires de l'Acad. des sciences de Paris, 1740) glaubte gefunden zu haben, dass die Nabelvene in die Aorta des Acardius mündet und nahm an, dass das lymphartige Blut, nachdem es durch die Nabelschnurvene in die Aorta und in die Capillaren des Acardius gelangt sei, aus diesen in das Zellgewebe ergossen, vielleicht auch theilweise durch die Haut ausgeschwitzt würde. Jedenfalls entstehe dadurch die starke Anschwellung des Zellgewebes.

Monro (Transactions of the Royal Society of Edinburgh, Vol. 3, 1794) liess das Blut durch die Nabelvene in den Acardius und durch die Nabelarterien zurück zur Placenta laufen.

J. F. Meckel (Handbuch der path. Anatomie, Leipzig 1812) neigte ebenfalls dieser Meinung zu, und hält den umgekehrten Weg für weniger wahrscheinlich.

Tiedemann (a. a. 0.) liess das Blut allerdings durch die Nabelschnurarterien zum Acardius und durch die Nabelschnurvene zur Placenta zurückströmen. Er fand aber das Movens für solche Bewegung in den Uebergangsstellen von den Nabelschnurarterien zu den Arterien des Acardius und von den Venen des Acardius in die Nabelschnurvene - "ähnlich wie in dem analogen Verhalten des Gefässsystems bei Mollusken, Fischen und im Pfortaderkreislaufe bei den Säugethieren und den Vögeln".

Emmert (1820) hat den Blutlauf in den Nabelschnurgefässen als der Norm entgegengesetzt erkannt.

Elben (De acephalis sive monstris corde carentibus. Dissert. Berol. 1821) hat die Literatur und die bis dahin gemachten Beobachtungen (72 Fälle) nochmals sorgfältig zusammengestellt und als erster das Fehlen des Herzens als gemeinsames Hauptmerkmal und als die Ursache der übrigen Körperdefecte dargelegt. Ueber die Art, wie die Circulation des Blutes stattfindet, hatte er jedoch nur sehr unvollkommene Vorstellungen.

Aber auch Marshall Hall (Edinb. and Lond. Monthly Journal XXX, p. 541) liess das Blut durch die Propulsivkraft des Herzens des gesunden Zwillings von der Placenta durch die Nabelschnurvene bis zum Acardius getrieben und rückwärts vom Acardius durch die Nabelschnurarterien desselben zur Placenta angesogen werden; und

Gurlt (Magazin f. d. gesammte Thierheilkunde 1840, VI. Jahrgang) stellt den Gefässkreislauf der Acardii nach dem Vorgang von Wedemeyer (Untersuchungen über den Kreislauf des Blutes 
etc. Hannover 1828) noch ganz analog dem Pfortaderkreislauf der Säugethiere dar, und bedachte nicht, dass das Blut dann beim Acardius ohne neuen Motor nicht bloss zwei, sondern sogar drei Capillargefässsysteme zu durchströmen hätte.

\section{Die neueren Autoren über die Circulationsverhältnisse der Acardii.}

Allerdings stellten schon Astley Cooper und Hodgkin (The history of an unusually formed placenta and imperfect foetus by Dr. Hodgkin, with an account of the structure of the placenta and foetus by Sir A. Cooper), welche die Anastomosen auf der Placenta erkannten, den Blutkreislauf in ihrem Acardius ganz richtig als in gleicher Weise wie in einem Glied des gesunden Kindes erfolgend dar.

Auch H. Neckel (Archiv für Anatomie ete. von J. Müller, 1850) liess den Kreislauf in derselben Weise erfolgen. Er erkannte sogar noch weiter richtig, dass nicht alle Acardii auf diese Weise entstehen und nahm an, dass sie theilweise auch aus einer allmäligen Selbstlösung von Parasitenbildung, oder dadurch entstehen, dass bei Vorhandensein eines doppelten Fruchthofes unter unbekannten Bedingungen die eine Area in der Entwicklung stehen bleibe, und der ihr entsprechende Fötus sehliesslich als blosser Anhang des anderen erscheine, dem ein besonderes Herz fehlt.

Aber dieser Fortschritt in der Erkenntniss, dass es mehrere ganz verschiedene Entstehungsweisen der Acardii mit entsprechenden Circulationsverhältnissen gäbe, brachte wegen seiner unbestimmten Fassung zunächst noch keinen wesentlichen Nutzen.

Hempel (De monstris acephalis. Dissert. Hafniae, 1850) kennt nur die eine Art der Acardiusbildung. Für diese spricht er aber zuerst mit aller Sicherheit aus, dass die Circulation bei allen Acardiis vermittelst der Anastomosen zwischen den Umbilicalgefässen des gesunden und denjenigen des herzlosen Zwillings in der Weise unterhalten werde, dass das Blut ron der Placenta zum Acardius durch dessen Nabelarterien und vom Acardius zur Placenta durch dessen Nabelvene fliesse.

Freilich drang auch Hempel mit seiner Darlegung noch nicht genügend durch. Z. B. gab

Förster 1855 (Lehrbuch der pathol. Anatomie, Wien) immer noch die Nabelschnurvene als das zu-, und die Nabelschnurarterie als das abführende Gefäss des Acardius an und erst 
Claudius (Die Entwicklung der herzlosen. Missgeburten, Kiel, 1858) fand in Betreff der Umkehr des Blutes in den Nabelschnurgefässen des Acardius allgemeine Zustimmung, so dass mit ihm wenigstens diese Frage als endgültig entschieden angesehen wurde. Freilich konnten Einzelne sich noch immer nicht von der Richtigkeit dieser Lösung überzeugen (s. Sitzungsbericht der Royal Medical and Chirurgical Society of London, Lancet. 1863, I. p. 580 und

Meïmaroglu, Dissert. Halle 1879, Ueber einen Acardiacus, - siehe auch unter „Umgrenzung des Begriffes" -).

Claudius begnügte sich aber nicht mit der Darlegung des Blutlaufes, sondern gründete darauf zugleich eine Hypothese der Aetiologie der Acardii überhaupt; und brachte damit diese weitere Frage so in Fluss, dass sie seitdem nicht mehr aus den entwicklungsgeschichtlichen Tagesfragen geschwunden ist (s. unten).

\section{Die neueren Autoren über die Aetiologie der Acardii.}

Gurlt hatte es noch 1840 (Magazin für die gesammte Thierheilkunde, VI. S. 5) sehr wahrscheinlich gefunden, dass die mangelhafte Bildung der Acardii schon bei der Anlage in der Keimhaut stattfindet, dass nämlich der vordere Theil der Rückenseite und der Rückenplatten, aus welchen sich der Kopf und der vordere Theil des Rumpfes bilden, bei den kopflosen Missgeburten sich gar nicht entwickelt. Es war ihm nicht wahrscheinlich, dass die hier fehlenden Theile schon einmal vorhanden gewesen und erst während des Fötuslebens wieder verschwunden wären; "es würden sich dann doch Spuren ihres früheren Daseins finden, wie wir sie bei verschiedenen Organen der normalen Fötusbildung, welche nur eine gewisse Zeit dauern und dann völlig verschwinden, kennen. Da nun die vordere Körperhälfte ursprünglich fehlt, so muss auch das Herz und der ganze Athmungsapparat fehlen. Die vorhandenen Blutgefässe sind daher eigentlich nur Verlängerungen der Nabelgefässe" etc.

1850 aber hatte, wie eben schon erwähnt, H. Meckel (Ueber die Verhältnisse des Geschlechts, der Lebensfähigkeit und der Eihäute bei einfachen und Nehrgeburten, Müller's Archiv 1850, S. 234ff.) zwei Arten der Entstehung der Acardii angegeben beide freilich nicht genügend klar und nicht genügend begrenzt, um gleich richtig und allgemein verstanden und anerkannt zu werden.

1. Es sollte bei Vorhandensein eines doppelten Fruchthofes 
unter unbekannten Bedingungen eine Area in der Entwicklung stehen bleiben und der ihr entsprechende -- von Anfang an herzlose - Fötus schliesslich als blosser Anhang des anderen erscheinen.

2. Meckel hatte bei mehrfacher Schwangersehaft sowohl das Absterben, wie auch die Monstrosität eines Fötus als häufig vorkommend gefunden (S. 239) und meinte, es "mögen bedeutende monströse Zustände des Fötus, namentlich Circulationstörungen durch Herzfehler und Ectopia cordis oft das Absterben bedingen, wenn nicht ein gesunder Zwilling vorhanden ist, der durch Placenta-Anastomose seiner Gefässe mit denen des kranken Fötus denselben auf seine Kosten erhält, wie bei den herzlosen Missgeburten.

H. Meckel kannte also sowohl die primären parasitären Acardii als auch die freien, in Folge von primärem Herztod entstehenden Acardii (s. unten Gruppe I).

Bezüglich letzterer rechnet H. Meckel (S. 245) etwa eine Monstrosität auf 3000 Geburten.

Devergie und Hohl (Geburten kranker, missgestalteter und todter Kinder. 1850. S. 164) geben tabellarische Zusammenstellungen über die Lebensfähigkeit der Monstra und sagen ebenfalls: „Sehr häufig sterben Acephalen, Anencephalen, Sirenenbildungen, Doppelmissgeburten schon im 9. und 8. bis 5. oder 4. Monat der Schwangersehaft ab."

Nach H. Ileckel (S. 451) sind die Doppelbildungen weiblichen Geschlechts weit häufiger zwillingssymmetrisch als die männlichen Geschlechts. Haller fand unter 42 symmetrischen Doppelbildungen nur 9 männliche, J. E. Meckel unter 80 Fällen 20 männliche, Otto unter 142 Fällen 52 männliche, Burdach nnter 258 Fällen 164 weibliche symmetrische, 17 weibliche unsymmetrische, 59 männliche symmetrische und 28 männliche unsymmetrische. Bei Acephalen ist ebenfalls das männliche Geschlecht überwiegend.

"Das im männlichen Geschlecht häufigere Vorkommen von Doppelbildungen, wo der eine auf Kosten des anderen mehr oder weniger zu Grunde ging, hängt mit der allgemeinen Erscheinung zusammen, dass zwei Brüder seltener einen gleichmässigen Gang der Entwicklung einhalten als zwei Schwestern. Von zwei in einem Amnion eingeschlossenen Früchten geht bei männlichem Geschlecht häufiger der Eine zum Vortheil des Anderen als Acephalus zu Grunde als bei weiblichem."

Dareste, welcher in Vogeleiern experimentell Missbildungen erzeugte, fand in Einlingseiern Embryonen, welche dem Acardius wenigstens ähnelten. Solche Embryonen müssen als Einlinge bald absterben und können nur als Zwillinge in Form des Acardius weiter existiren. Dareste sagt (Recherches sur la production artificielle des monstrosités 1877, 
p. 312, p. 316): "L'absence du coeur a done pour résultat d'arreter à un certain moment l'évolution de l'embryon, et d'amener rapidement sa mort à moins qu'elle ne soit compensée par l'intervention d'une condition physiologique particulière, la gémellité.

Or il résulte de nos recherches, que si la gémellité joue un grand rôle dans l'existence de monstres omphalosites à une certaine époque de leur vie, elle est entièrement étrangère à leur origine. Les monstres omphalosites peuvent se constituer isolement, comme les monstres autosites, mais ils ne peuvent continuer à vivre que dans le cas de la gémellité."

Dareste kennt also nur primäre Acardii, die nur parasisitäre sein können, d. i. die 1. Art von H. Meckel.

Aehnlich wie Dareste spricht sich Panum aus. (Beiträge zur Kennitniss der physiologischen Bedeutung der angeborenen Missbildungen. Virchow's Archiv 72. Bd. 1878.) Er sagt: „Meine Untersuchungen über die Entstehung der Missbildungen, zunächst in den Eiern der Vögel, haben mir gezeigt, dass diejenigen Missbildungen, bei welchen eine Umgestaltung der ganzen Körperform oder eine Molenbildung vorhanden ist, in der allerersten Periode der Entwicklung durch Erkrankung (Entzündung) der Anlage des Embryos entstehen, und dass man durch Ablühlung und äussere Schädlichkeiten, welche das Ei während der drei ersten Tage der Bebrütung treffen, in der Regel solche Molenbildung oder Zerstörung der ganzen Körperform hervorbringt, während dieselbe Ursache auf einem späteren Stadium mehr localisirte Bildungsfehler der gerade in ihrer ersten Ausbildung befindlichen Organe bewirkt. Ich habe demnächst auch beim Menschen den in den Vogeleiern beobachteten ganz ähnliche Molenbildungen beschrieben und abgebildet, welche in Abortiveiern gefunden waren. Solche Molen werden begreiflicherweise, wenn sie allein im Uterus vorhanden sind, schon früh absterben und abortiv zu Grunde gehen und ausgestossen werden, weil die Herzthätigkeit und Blutbereitung in einem so ganz missgestalteten und verkrüppelten Embryo ohne Zweifel sehr bald zur Ernährung desselben ungenügend werden muss. Ganz anders gestalten sich aber die Verhältnisse, wenn in einer solchen Mole durch Vermittlung eines gesunden, gleichzeitig im Uterus entwickelten $Z$ willings, ein Kreislauf des brüderlichen Blutes unterhalten werden kann. In solchem Falle kann selbst nach sehr umfangreichen Zer- 
störungen und Defecten ein ganz verkrüppelter Rest eines Embryo im Uterus noch bis zur normalen Zeit der Geburt hin entwickelt werden".

Alle drei Autoren H. Meckel, Dareste und Panum nehmen also als Entstehungsursache der Areadii primären Defect oder primären Tod des Herzens an, welcher verursacht oder mindestens begleitet ist von primären Defecten oder Missbildungen des ganzen Embryo.

Diese Vorstellungen haben viele Anhänger gefunden. Z. B. nimmt: Perls (Allgem. Aetiologie der Missbildungen, 1879, S. 322) an, dass für die Entstehung der Acardii nicht die Claudius'sche Erklärung (s. unten), sondern „anderweite Momente, wie Einschnürung durch Eihäute und Nabelschnur die Hauptrolle spielen, und dass in den meisten Fällen hochgradiger Verstümmelung der ganze Fötus abstirbt und zu Grunde geht, dass aber in den Fällen von Zwillingsschwangerschaft der mit der Nabelschnur in Verbindung bleibende Theil durch die Anastomose mit der Nabelschnur des normalen Zwillings genügende Blutzufuhr bekommt, um sich weiter entwickeln zu können".

Auf demselben Standpunkt stehen mit geringen Abweichungen Breus (Zur Lehre von den Acardiacis, Wiener med. Jahrbücher 1882) und Andere.

Gegenüber dieser Hypothese (welche ich nach ihren Urhebern die Meckel-Dareste-Panum'sche oder auch kurz die Meckelsche nennen will) hat Claudius (Die herzlosen Missgeburten. Kiel 1859) eine deutlich abgegrenzte entgegengesetzte aufgestellt.

Claudius giebt wörtlich folgende Erklärung:

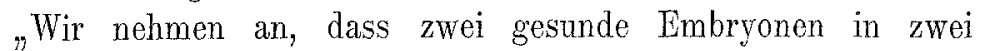
dicht neben einander liegenden Eiern zur normalen Zeit ihre Allantojden nahe an einander an die Innenseite ihrer Chorien ansetzen, dass auf den sich entwickelnden Placenten zwei Arterien zufällig gerade einander entgegenwachsen, sich berühren und endlich in einander öffnen; die sie begleitenden Venen thun dasselbe. Was muss nun die nächste Folge eines solchen Uebergangs von einer Nabelarterie in die andere sein? Die von jedem der beiden Herzen fortgeschleuderten Blutwellen müssen in der Mitte des Weges aufeinanderstossen, ihr Fortschreiten hemmen, und so eine Rückstauung des Blutes gegen das Herz hin veranlassen."

${ }_{n}$ Findet nämlich bei gleicher Gefässstructur in beiden Herzen 
in demselben Augenblick eine Systole mit gleicher Kraft statt, so müssen die beiden Blutwellen in der Mitte des Weges zusammentreffen, hier eine Interferenzwelle bilden und dann in der früheren Richtung unverändert fortschreiten. Geschieht eine der beiden Systolen nur mit der Hälfte der Kraft, womit das andere Herz sich zusammenzieht, so erlischt die erstere Welle in dem Interferenzpunkt und die andere schreitet mit der halben Pulshöhe fort. Während der kurzen Zeit eines solchen Kampfes trifft nun die Systole des einen Herzens in tausendfachem Wechsel mit allen Zeitpunkten, die zwischen zwei Systolen des anderen Herzens liegen, zusammen, und wenn alle Verhältnisse gleich wären, so müssten beide Herzen, «die sich beständig aneinander entgegen arbeiten, endlich vor Kraftersehöpfung stille stehen (?!). Dieses ist aber wohl nie der Fall; einmal ist der eine von zwei Föten gewöhnlich etwas weniger gut entwickelt, und zweitens wird leicht der Zufall in dem Zusammentreffen der Pulswellen dem einen eine grössere Kraftäusserung auf das Herz des anderen gestatten. Sobald aber das eine Herz sich mit geringerer Energie contrahirt, als das andere, ist es im entschiedenen Nachtheil; es hemmt das andere Herz weniger und wird direkt viel stärker gehemmt. $\mathrm{Ob}$ gleich die Pulswelle, welche aus dem Herzen der gesunden Frucht durch die Nabelarterie des späteren Acardiacus eintritt, in der weiteren Aorta viel von ihrer Kraft verliert, so wird sie doch die aus dem Herzen des letzteren ihr entgegenkommende schwächere hemmen, oder bei einem Kraftüberschuss sich durch sie hindurch aufwärts fortpflanzen. Das Endresultat muss ein Stillestehen des einen Herzens sein."

"Es sind dies hämatodynamische Verhältnisse, wie sie wohl in dem ganzen Reich der thierisehen Natur schwerlich ihres Gleichen haben. Auch können wir uns nicht leicht einen deutlichen Begriff von dem Vorgang beim Tode des Herzens machen (!). Wenn in dem Moment der Systole eines Herzens eine Blutwelle mit derselben Kraft gegen die Semilunarklappen, oder, da diese zu der Zeit schwerlich schon sufficient sind, in beide Ventrikel schlägt, so wird die Zusammenziehung derselben unmöglich; der übermässigen Kraftanstrengung muss eine Lähmung folgen. Am ehesten können wir diesen Vorgang wohl jenem vergleichen, der auf Luftzutritt in das venöse Herz erfolgt. Das mechanische Hinderniss der erwärmten Luft verursacht Stillstand." 
Nachdem auf diese Weise (in der Vorstellung von Claudius) das Herz des späteren Acardius getödtet ist, wird die Circulation in diesem nur von dem gesund gebliebenen Mitzwilling besorgt. Da dieselbe theils umgekehrt, theils vielfach ungenügend ist, wird der Acardius in seiner ganzen Entwicklung gehemmt, und bei der Ungleichheit der Ernährung in der verschiedensten Weise verstümmelt, meist so, dass die untere Hälfte des Körpers am meisten ausgebildet ist, weil dort die Nabelschnurarterien, welche das ernährende Blut bringen, eimmünden.

Diese Claudius'sche Hypothese, welche die Aetiologie der Acardii unter dem einheitlichen Gesichtspunkt der Placentaanastomosen betrachtete und von ihnen zuerst den Ferztod und erst secundär die Verstümmelung des Acardius ableitete, fand vor der Meckel'schen Hypothese, welche zweier Momente zur Erklärung bedurfte - neben den Placentaanastomosen noch primäre Missbildung des späteren Acardius - erklärlicher Weise vielen Anklang und war eine Zeit lang fast überwiegend, z. B. auch von Poppel ${ }^{1}$ ) angenommen. Die physiologischen - - um nicht geradezu zusagen physikalischen - Schwächen derselben waren aber doch zu gross, als dass sie sich hätte lange behaupten können.

Claudius hatte zwar gleich in seiner ersten Arbeit selbst eine gewisse Einschränkung seiner Hypothese eingefügt und S. 51 bemerkt: "dass nicht jede Anastomose zweier von verschiedenen Herzen kommenden Arterien eine Rückstauung des Blutes aus der einen in die andere nothwendig zur Folge haben muss. Wenn zwei dicht neben einander parallel laufende Arterien zweier Früchte in einem Nabelstrang sich in eine hinreichend weite gemeinschaftliche Arterie öffnen, so können beide Früchte gesunde Herzen behalten, weil hier aus begreiflichen Gründen (?!) die Pulswelle einer Arterie sich nur mit äusserst geminderter Kraft rückwärts in die andere fortpflanzen kann. Hierher gehört ein von Reynolds in

1) Poppel (Monatsschr. f. Geburtsh., XX., S. $251 \mathrm{ff}$.) nimmt ebenfalls an, dass zwei Arterien von beiden Seiten aufeinander treffen und anastomosiren können. Darans entstehe bei gleichem Druck Stauung und Obliteration. Aber ,gewiss in den meisten Fällen giebt die Anastomose dadurch zur Entstehung eines Acardiacus Veranlassung, dass bei constant etwas überwiegender Propulsiviraft des Herzens der einen Frucht der Blatstrom dieser die Oberhand gewinnt und den der anderen bis zurück zum Herzen staut und endlich in umgekehrter Richtung zu fliessen zwingt; dies muss nothwendig anfänglich Unregelmässigkeit und bald Lähmung der Herzcontractionen der überwundenen Frucht zur Folge haben. Das Herz geht dann atrophisch zu Grunde." 
einer amerikanischen Zeitschrift veröffentlichter, in Julius und Gerson, Magazin für ausländische Literatur, 1835, kurz excerpirter Fall von Zwillingen, die ein langes Stück der Nabelschnur mit Einer Arterie und Einer Vene gemeinschaftlich besassen. Beide seheinen gesund gewesen zu sein. Eine Verklebung beider Allantoiden muss die Ursache dieser Verschmelzung gewesen sein."

In späterer Zeit machte Claudius nach einer Angabe in der Dissertation von Eysell (1867, S. 19) sogar noch weitere Concessionen. Er gab einen primären Herztod des späteren Acardius zu, und hatte die Ansicht, dass aus den wahrscheinlicb früh zwischen den feinsten Gefässen in den Chorionzotten ausgebildeten Communicationen eine weite Anastomose werde, wenn der Foetus durch eine andere Ursache absterbe. "Es wird sich wahrscheinlich derselbe Vorgang entwickeln, wie bei Unterbindung eines Arterienstammes im erwachsenen Körper. Es entwickeln sich zuerst eine ganze Anzahl von Collateralästen. Nach und nach rinnt dureh eine Bahn, die die bequemsten hydrodynamischen Verhältnisse bietet, der ganze Blutstrom, während die anderen Aeste sich zurückbilden und verschwinden. - Hiernach könnte also der Tod der einen Zwillingsfrucht das Primäre und die Anastomose auf der Placenta das Secundäre sein."

Solche Concessionen zeigten aber erst recht, dass die Hypothese von Claudius auf sehr schwachen Füssen stand. Sie musste dem Andrängen der Anhänger der Meckel-Dareste-Panum'schen Hypothese immer mehr weichen, sodass Ahlfeld glaubte, ihr durch eine Revision zur Hülfe kommen zu müssen.

Diese Revision bezieht sich hauptsächlich auf den Ort und die Ursache, wo und wodurch zuerst die Umkehr des Blutlaufes stattfindet.

Ich will, um grössere Wiederholungen zu vermeiden, die etwas umständlicheren Auseinandersetzungen der Ahlfeld'schen Revision erst bei der Kritik derselben geben.

\section{Kritik der neuen Hypothesen.}

Die oben in der Literatur aufgezählten Hypothesen bis zu Tiedemann bedürfen, weil von Niemandem mehr gehalten, in der Jetztzeit einer kritischen Widerlegung nicht mehr. Es bleiben also nur die beiden Hypothesengruppen von Meckel-Dareste-Panum und von Claudius-Ahlfeld zu besprechen übrig. Beide könnte ich sehr kurz dadurch abthun, dass ich zeigte, dass sehr viele 
Acardii durch sie nicht zu erklären sind. Doch erscheint es mir zweckmässig, sie etwas genauer zu besprechen, weil dadurch zugleich das Verständniss für meine eigene Hypothese erleichtert wird.

Beide Hypothesengruppen haben als grössten Fehler gemeinsam, dass sie die Frage von einem zu einseitigen Standpunkte, allerdings jede von einem anderen aus, betrachten, sie von ihm allein aus lösen wollen und nicht bedenken, dass die Natur, wie so oft, eine ganze Anzahl verschiedener Wege hat, auf welchen sie entweder wirklich oder wenigstens scheinbar zu demselben Resultat kommt.

Am umsichtigsten verfährt noch $\mathrm{H}$. Meckel, indem er, wie wir sahen, zwei deutlich verschiedene Entstehungsarten der Acardii unterscheidet:

1. primäre - durch Nichtentwickelung des Herzens in dem einen der beiden Fruchthöfe eines Zwillingspaares und

2. secundäre - durch Tod eines Zwillings in Folge von Missbildungen, besonders des Herzens.

Die übrigen Autoren sind viel einseitiger. Dareste kennt fast nur die primäre Acardie durch Nichtentwickelung des Herzens ( 1 bei H. Meckel) und Panum will, wenn er auch ein Herz vorhanden sein lässt, das Absterben desselben stets durch Missbildung des betreffenden Zwillings bedingt wissen ( 2 bei $\mathrm{H}$. Meckel). Claudius will umgekehrt nur normale Früchte zu Acardii werden lassen und arbeitet dabei eigentlich nur mit der arteriellen Anastomose und mit dieser noch dazu in einer gar zu einfachen und unglaublich unphysikalischen Weise. Man muss sich wundern, dass seine Hypothese überhaupt so vielseitig angenommen worden ist. Es liegt das wahrscheinlich nur daran, dass wenigstens der zweite Theil seiner Hypothese einiges Wahre enthält. Es war nutzlos, dass Ahlfeld die unphysikalische Hypothese von Claudius durch mehrseitige eigene Zuthaten stiitzen und halten wollte. -

Für die übrigen Einwände sind die beiden Hypothesengruppen getrennt zu behandeln.

\section{Kritik der Hypothese von Meckel-Dareste-Panum.}

Spricht man von Acardie ganz im Allgemeinen, so muss man allerdings anch alle die Fälle einschliessen, wo der eine der beiden Fruchthöfe eines Zwillingseies kein Herz entwickelt oder dasselbe absterben lässt, so dass der gemeinsehaftliche Dotterkreislauf den herzlosen Fruchthof vom Herzen des gesunden Fruchthofes aus mit 
unter Circulation hält und ernährt. Das ist wirkliche primäre Acardie.

Solche Acardii giebt es auch ziemlich häufig. Sie sind aber stets ohne Nabelschnur und parasitisch mit dem Hauptzwilling verbunden. Indem Meckel, Dareste und Panum solche Acardii beschreiben, schildern sie nicht die Entwickelung der Acardii, sondern nur eine Art der Entwickelung von Acardii. Sie hätten dies ausdrücklich sagen müssen, wollten sie vor Missverständnissen bewahrt bleiben. Dadurch, dass Panum sogar weitere Fälle mit heranzog, bei welchen auch nach Einleitung des Placentakreislaufes Missbildung des einen Zwillings diesen unter Mithülfe des gesunden Zwillings zum Acardius machte, musste das Missverständniss noch grösser werden. Meckel schied zwar, wie oben gezeigt ist, die mit Nabelschnur versehenen - freien - Acardii, welche für gewöhnlich allein als Arcadii bezeichnet werden, deutlich ab. Sie spielen aber auch bei ihm zu sehr eine Nebenrolle. Dazu kommt, dass er unter ihnen fast nur die Fälle mit primärem Herztod berücksichtigt. Er lässt dadurch den grössten Theil aller Acardii unerklärt. Soweit die Hypothese von H. Meckel, Dareste und Panum sich auf die primäre Entstehung der Acardie bezieht, soll sie entsprechend der weiter unten gegebenen Ungrenzung meines Themas hier nicht weiter kritisirt werden. Die so entstehenden Acardii sind eben parasitär, ohne Nabelschnur und gehören nicht hierher.

Was von der Hypothese für die freien Acardii übrig bleibt, ist fast nur noch die Forderung, dass der künftige Acardius von Anfang an missgebildet gewesen sein soll, so dass er entweder von selbst abstirbt oder wenigstens das Herz des gesunden Zwillings leichte Arbeit hat, dasjenige des missgebildeten zu unterdrücken. In beiden Fällen kann bei Vorhandensein genügender Placentaanastomosen der abgestorbene Zwilling vom überlebenden mehr weniger vollkommen weiter ernährt werden.

So gefasst ist diese Hypothese bestechend und scheint eine weitere Begründung in der Häufigkeit zu haben, mit welcher gerade bei Zwillingsschwangerschaft Missbildungen vorgefunden werden. Es ist auch ganz zweifellos, dass auf diese Weise Acardii entstehen. So finden wir z. B. unter den Acardiis ungewöhnlich häufig partiellen Situs transversus der Gefässe. Partieller Situs transversus disponirt besonders zu Missbildungen. Ein im Ganzen oder wenigstens in seinem Gefässsystem missgebildeter Fötus wird leichter 
absterben, oder es wird wenigstens sein. Herz leichter überwunden werden.

Diese Hypothese kann aber nur für einen Theil und durchaus nicht für alle oder auch nur für die Mehrzahl der Acardii richtig sein. Denn:

1. Wir kennen selbstständige und mit Nabelschnur versehene Acardii, die, abgesehen von Fehlern des Herzens und der direct davon abhängigen Organe, Missbildungen gar nicht zeigen. Nun könnte man allerdings annehmen, dass in solchen Fällen primär eben nur das Herz missgebildet war. Es ist aber doch schwer glaublich, dass bei ihnen gerade das Herz am unvollkommensten gebildet gewesen sein soll - das Organ, welches bei der Allantoissprossung doch die Hauptthätigkeit entwickelt haben muss.

2. Es mögen die Missbildungen bei eineiigen Zwillingen relativ noch so häufig sein und diese mögen auch oft in Folge dessen absterben und dann zu Acardiis werden: Unter der Gesammtzahl der abgestorbenen eineiigen Zwillinge sind sie doch nur ein sehr kleiner Theil und unter den eineiigen Zwillingsschwangerschaften, bei welchen der eine Zwilling abstirbt, finden wir diesen im Ganzen viel seltener missgebildet, ungleich häufiger normal gebildet. Es wäre auffällig, wenn die Acardii sich gerade nur aus der geringen Zahl der abgestorbenen missgebildeten Zwillinge rekrutiren sollten. Es lässt sich nicht einsehen, warum bei sonst ganz gleichen Verhältnissen ein absterbender normaler Zwilling nicht ebenso gut vom überlebenben Mitzwilling weiter mit ernährt werden soll wie ein absterbender missgebildeter. Schon danach müsste wenigstens eine grosse Anzahl der Acardii aus normal gebildeten Früchten entstehen.

3. Die verschiedenen Formen, welche die Acardii bieten, stimmen in ihrer relativen Häufigkeit durchaus nicht mit den verschiedenen sonst bei Missbildungen eineïger Zwillinge gefundenen Eormen überein. Wenn man nun auch zugeben will, dass sich nur bestimmte Missbildungen besser zur Entstehung von Acardie eignen werden als andere, so sind doch wieder viele Formen der Acardii bei den sonstigen Missbildungen gar nicht vertreten, z. B. die Acormi, manche Formen der Amorphi, ja auch der Acephali. Die Acardii bieten also, so sehr sie auch Formen annehmen können, die auch sonst vorkommen, immer noch eine ganz besondere, mit charakteristischen Merkmalen versehene Reihe von Missbildungen, welche sich mit derjenigen der gewöhnlichen Miss- 
bildungen weder deckt, noch ihr parallel läuft und welche durch die Gesetze der sonstigen Missbildungen auch dann nicht vollständig erklärt werden kann, wenn man die Defecte hinzunimmt, welche durch Eintritt der Acardie hinzukommen.

4. Claudius hat schon richtig bemerkt (a. a. O. S. 26): "Der Foetus muss bis zur Zeit der Bildung der Placenta und der Anastomosen mit den Gefässen des Zwillingsfoetus ein vollkommen funktionirendes Herz gehabt haben, weil sich eine normale Allantois, ein normaler Nabelstrang und anfangs eine normale Placenta gebildet haben."

Später fehlt das Herz - wenigstens sehr häufig - ganz. Wenn dieses Organ, welches bis zum Eintritt der Acardie das Wichtigste und am sichersten vorhanden war, später ganz verschwindet, so können und werden wohl auch die anderen Organe, welche später fehlen, ursprünglich vorhanden gewesen sein. Die Defecte der sonstigen Organe sind sicher nicht schwerer zu erklären, als derjenige des Herzens. Es liegt also gar keine Nöthigung vor am Acardius überhaupt primäre Defecte anzunehmen: H. Meckel hat dies auch anerkannt, indem er das häufige Absterben eineiiger Zwillinge - ohne Missbildung - als genïgende Ursache für Acardie mitnannte.

Wird aber die Bedingung der primären Missbildung von der Meckel-Dareste-Panum'schen Hypothese fortgenommen, so bleibt von dieser gar nichts übrig. Dareste und Panum haben denn auch diese Bedingung nicht fallen lassen, obgleich sie durch H. Meckel genügend darauf aufmerksam gemacht waren.

5. Die Hemiacardii finden bei der Meckel-Dareste-Panumschen Hypothese nur gekünstelte Erklärung. Bei ihnen müsste die Missbildung des Herzens auch primär, aber doch nicht gross genug sein, um den Tod des Herzens zu bewirken.

6. Einzelne Formen der Acardii sind durch die MeckelDareste-Panum'sche Hypothese überhaupt nicht zu erklären $z$. B. -diejenigen mit Fortbestand der Dottervene neben den Allantoisgegefässen.

7. Die Hypothese wird auch bei den gewöhnlichsten Formen als ganz einseitig und ungenügend erkannt, sobald man auf feinere Processe bei Entstehung der Acardie, auf die gesammten Gefässverhältnisse und die Defecte am Acardius näher eingeht.

Trotz aller dieser Gegengründe fasst einer der neuesten Autoren auf diesem Gebiete, Breus (Wiener Medicinische Jahr- 
bücher. 1883. I. S. 57), welcher der Claudius-Ahlfeld'schen Hypothese energiseh widerspricht, aber nach dem Vorgang von Perls (Pathologische Anatomie) den Vorstellungen von Dareste und Panum zustimmt, seine Meinung dahin zusammen: "Acardiaci sind durch frühzeitige Störung ihrer embryonalen Entwicklung so hochgradig defecte Früchte, dass sie einer selbstständigen Circulation und intrauterinen Ernährung gar nicht fähig waren, die aber trotzdem sich noch weiter entwickelten und fortwucherten, weil eine supplirende Circulation ihre Gewebe lebend und wachsend erhielt."

Zu dieser Erklärung passt aber gleich der eigene Fall von Breus, welcher die. Veranlassung zu dieser Erklärung gab, durchaus nicht, denn dieser Acardius ist sehr vollkommen. Thm fehlt nur das Herz und das, was mit ihm gewöhnlich zu Grunde geht. Ausserdem kann die Entwickelungsstörung bei ihm gar nicht in so früher Entwicklungszeit eingetreten sein, weil die Nabelschnur gut entwickelt war.

\section{Kritik der Hypothese von Claudius.}

Claudius hat zur Grundlage seiner Hypothese den Satz gemacht, dass der Blutlauf in den Nabelschnur- und den entsprechenden grossen Körper-Gefässen (Hypogastricae und Aorta) des Acardius stets umgekehrt ist. Diese Entdeckung rührt zwar nicht von Claudius, sondern von Hempel her. Doch hat erst Claudius verstanden daraus allgemeinere Folgerungen zu ziehen daraus ein bestechendes System aufzubauen. Diese Grundlage der Hypothese ist im Allgemeinen auch richtig. Ihre allgemeine Gültigkeit erfährt zwar durch die vorliegende Arbeit eine gewisse Einschränkung, indem ich zeige, dass neben den Holoacardiis, welche volle, und neben den Hemiacardiis, welche wenigstens theilweise Umkehr des Blutlaufs (zwischen Placenta und Herz) zeigen, noch eine Reihe von Bildungen existirt - die Pseudohemiacardii - welche den Hemiacardiis ganz analog und ihnen ähnlich geformt sind, auch ein ebenso verkümmertes Herz haben, bei welchen aber keine Umkehr des Blutiaufs statt hat. Diese ganz bestimmte und umgrenzte Ausnahme, welche in Gruppe III eingehend erörtert werden soll, ändert aber an der Richtigkeit des Hempel'schen Satzes von der Umkehr des Blutlaufes bei den Acardiis nichts und wenn sie auch noch 1863 z. B. in der Discussion über den Fall Dickinson (Lancet. 1863. I. S. 580) von mehreren Seiten 
bestritten worden ist und wenn Meïmaroglu (Diss. Halle) sogar noch 1879 bei seinem Fall den normalen Lauf des Blutes in den Nabelschnurgefässen beweisen zu können glaubt, so werde ich doch letzteren Fall weiter unten kritisch zurückweisen und in der Discussion über Dickinson's Fall konnte auch kein beweisendes Moment für die abweichende Meinung angeführt werden.

Ebenso richtig ist an der Hypothese von Claudius die Auffassung, dass die Acardii im Allgemeinen ursprünglich normale oder nur wenig abnorme Früchte sind und dass ihre späteren Defecte und Missbildungen wenigstens in der Hauptsache erst durch die Umkehr des Blutlaufes nach Eintritt der Acardie erzeugt werden. Freilich hat Claudius sich die Wirkung des umgekehrten Blutstromes zu einfach gedacht, worüber weiter zu sprechen sein wird. Alles andere aber von der Hypothese von Claudius ist falsch.

1. Zunächst kennt Claudius - wenigstens in seiner ersten bezüglichen Veröffentlichung - nicht die Entstehung von Acardii durch primären Herztod, welche schon H. Meckel gekannt und berücksichtigt hatte. Später gab Claudius allerdings auch diese Entstehungsweise zu.

2. Wollte man den Satz von Claudius festhalten, dass die Acardii wirklich nie ein Herz haben, so würde man die ganze Reihe der Hemiacardii ausscheiden müssen, und doch kann man sie als Uebergänge zum normalen Verhalten der Zwillinge gar nicht entbehren und auch deshalb nicht fortlassen, weil sie nicht allein schon theilweise Umkehr des Blutlaufes zeigen, sondern weil sie schliesslich zumeist sogar noch wirkliche Acardii werden, indem das gewöhnlich zwar unvollkommene, aber doch vorhandene Herz schliesslich doch noch ausser Thätigkeit gesetzt wird. Durch das Fortlassen der Hemiacardii hat Claudius seiner Hypothese einen grossen Theil ihrer Natürlichkeit genommen. Die Acardii kommen dadurch ganz ausser Zusammenhang mit den übrigen Formen der eineiigen Zwillinge - eine Isolirtheit, welche von vornherein nicht wahrscheinlich ist.

3. Am meisten aber hat Claudius seiner Hypothese geschadet durch die ganz unglaublich unphysiologische, ja unphysikalische Erklärung, welche er für die Umkehr des Blutlaufes gegeben hat.

Zunächst hatte Claudius keine Ahnung von den Circulationsverhältnissen vor und bei Beginn der Placentabildung. Fr lässt 
sich da eine grosse arterielle Anastomose neu bilden, wäbrend in Wirklichkeit zu dieser Zeit von den früheren massenhaft vorhandenen Anastomosen die meisten veröden und schliesslich nur die eine zurückbleibt.

Dann lässt Claudius die Herzen beider Zwillinge mittelst dieser Anastomose gegeneinander an arbeiten und das schwächere, unter ungünstigeren Verhältnissen arbeitende Herz vor der andrängenden Blutwelle des kräftigeren Herzens erlahmen und stillstehen, und bedenkt nicht, dass die scheinbar gegeneinander andrängenden Blutmassen in ihren ursprünglichen Bahnen - d. i. in ihrem eigenen vielverzweigten Choriongefässnetz - so viele Auswege haben, dass es in der Anastomose oder an irgend einer anderen Stelle zu einem besonders hohen Blutdruck gar nicht kommen kann. Fliesst aber bei Differenz des Blutdruckes an den beiderseitigen Enden einer Anastomose von der Seite des Zwillings mit höherem Blutdruck Blut über, so fliesst dies jenseits zunächst durchaus nicht dem dortigen arteriellen Strom entgegen, sondern nach der Richtung des geringeren Widerstandes mit ihm, also in der Richtung des dortigen Arterien-Blutes nach den Zotten hin. Es kommt damit in die jenseitigen Venen und bewirkt durch vermehrte Speisung des schwächeren Herzens in Wirklichkeit Kräftigung desselben, während umgekehrt Claudius durch das Ueberfliessen auf dem arteriellen Wege Stillstand desselben entstehen lassen will.

4. Claudius confundirt bei letzterer Vorstellung immer Blutwelle und Blutdruck.

5. Claudius lässt sich verleiten (I. c. S. 22, 23), die GefässverhäJtnisse an den fertigen Acardiusplacenten allgemein auch für die Zeit der Entstehung des Acardius gelten zu lassen. Dies ist nur angängig bei den relativ wenigen Fällen, wo der Acardius sehr spät solcher geworden ist. In allen Fällen, wo der Herztod schon früh eintritt - und diese bilden bei weitem die Mehrzahl -, wandelt sich die Placenta nach den neuen Verhältnissen so gründlich um, dass aus dem späteren Zustand der Placenta nur sehr wenige Schlüsse auf den früheren gemacht werden können (s. unten die "Placentaverhältnisse der Acardii“).

\section{Kritik der Hypothese ron Ahlfeld.}

Um die hauptsächlichsten der eben gerügten Schwächen der Hypothese von Claudius zu beseitigen, hat Ahlfeld bei seiner 
Revision derselben besonders Aenderungen nach zwei Richtungen vorgenommen.

a) Er hat den Ort, wo der Blutstrom zuerst umkehren soll, nicht wie Claudius in eine einzige Anastomose verlegt, sondern in die Gesammtheit der massenhaft vorhandenen capillären Anastomosen. Damit aber in diesen die Umkehr stattfinde, hat er

b) angenommen, dass der spätere Acardius von vornherein ein Allantois-Parasit sei, d. h. dass seine Allantois nicht oder nur unvollkommen auf die spätere Placentastelle der serösen Hülle auftreffe, so dass er, weil er der eigenen Ernährung entbehre, schwächer werden bez. bleiben müsse.

Diese beiden Aenderungen erregen freilich schon von vornherein Bedenken.

a) Die erste kann der Hypothese nichts nützen; denn die hydro- resp. haematodynamischen Gesetze sind, soweit sie hier in Anwendung kommen, in engen Röhren dieselben wie in weiteren. Das Blut läuft immer nach der Richtung des geringeren Druckes.

Wenn aber durch das Heranziehen der massenhaften Gefässe derjenige Vorgang verständlicher gemacht werden soll, durch welchen der Blutstrom des einen Zwillings den des anderen überwindet, so muss man einwenden, dass mit der durch die vielen Gefässe gegebenen grösseren Möglichkeit sowohl der Umkehr des Blutstromes nach den eigenen Venen hin, als auch des Ueberfliessens durch die Capillaren in die jenseitigen Venen hin (wie oben bei Claudius unter 3) die Wahrscheinlichkeit der Ueberwindung des fremden Blutstromes nur vermindert wird.

b) Die Annahme, dass derjenige Zwilling, dessen Allantois nicht auf einen freien Theil der serösen Hülle oder wenigstens nicht auf die spätere Placentastelle auftrifft, schwächer ernährt werden müsste, ist willkürlich.

Man kann nicht einmal zugeben, dass der Zwilling, dessen Allantois zu spät kommt und nicht mehr direct auf die seröse Hülle, sondern nur noch auf die schon ausgebreitete Allantois des ersten Zwillings auftrifft, nicht eine eigene Placenta bekäme. Die Natur weiss sich dabei sehr gut zu helfen.

Die beiden Allantoides bilden, mag die eine viel oder wenig zur Ausbreitung auf der serösen Hülle beigetragen haben, schliesslich immer ein durchaus gemeinschaftliches primäres Choriongefässnetz und hätte selbst die zweite Allantois gar nichts zu dieser Ausbreitung beigetragen, sondern wüchse sie einfach auf die schon 
ausgebreitete erste Allantois zu, so würde sie mit dieser ebenso innige Gefässverbindungen eingehen, wie es geschähe, wenn sie sich mit ihr schon wäbrend der Ausbreitung auf der serösen Hülle getroffen hätte. Das primäre Choriongefässnetz wäre immer wieder durchaus gemeinschaftlich und der zweite Zwilling würde sein Blut ebenso gut wio der erste in dasselbe hineingeben und daraus erhalten. Es würde sich das primäre Choriongefässnetz ebenso wie bei gewöhnlichem Verhalten der Allantoiden functionell in zwei Hälften theilen and es würde sich schliesslich auf der Eiinsertion -event. unter Verschieben des Circulationsäquators (s. oben Bd. XXVII, S. $43 \mathrm{ff}$.) - auch für den zu spät gekommenen Zwilling eine Placentahäfte bilden, obgleich dieser ursprünglich dazn gar keine Allantoisgefässe geliefert hat.

Ich besitze eine eineiige Zwillingsplacenta, bei welcher die Nabelschnur des einen Zwillings auf dem Rande der Placenta, die des anderen gerade gegenüber am anderen Pol des Eies in die Eihaut inserirt ist. Von dieser Stelle aus laufen die Gefässe getheilt - $16^{1} / 2 \mathrm{~cm}$ d. i. um einen vollen Quadranten des Bies - zu dem der Insertion der ersten Nabelschnur entgegengesetzten Rande der Placenta. Diese ist nach dieser Seite hin eine marginata. Die beiden Arterien und die beiden Venen, welche von der velamentösen Nabelschnurinsertion nach der Placenta laufen, senken sich in diese natürlich erst ein, nachdem sie über den chorialen Ring hinweg auf die eigentliche fötale (napfförmige) Placentaoberfläche angekommen sind.

Bei diesem Zwillingsei ist deutlich erwiesen, dass die Allantois des einen Zwillings nicht auf die Insertionsstelle des Eies (Serotina) aufgetroffen ist, sondern um wenigstens $90^{\circ}$ davon ab - am reifen Ei 161/2 cm vom nächsten Placentarand und $35 \mathrm{~cm}$ ron der Insertion der anderen Nabelschnur - so dass die beiden Nabelschnurinsertionen um $180^{\circ}$ von einander entfernt waren. Es kann hier auch nicht eingewendet werden, dass die velamentöse Insertion vielleicht nur seheinbar sei, indem die Strecke von der Nabelschnurinsertion bis zur Placenta verödete Placenta sein könne. Der Umstand, dass die Placenta auf dieser Seite marginata ist, beweist, dass die Insertion des Eies wenigstens nach dieser Seite hin nicht einmal so weit reichte wie die ausgebildete Placenta.

Hier hat also die primäre Ausbreitung der zweiten Allantois offenbar nicht bis zur späteren Placentastelle gereicht. Trotzdem ist die schliessliche Placenta ziemlich gleichmässig auf beide Zwil- 
linge vertheilt und beide Zwillinge waren gleich gut entwickelt, 43 bez. $431 / 2$ cm lang, $1 / 2$ bez. $3 / 4$ I Fruchtwasser.

Der Circulationsäquator zwischen beiden Allantoisgefässnetzen, welcher anfangs gar nicht oder höchstens am Rande über die Serotina lief, wurde bis nahezu in die Mitte derselben verschoben und die dann noch gebliebene Ungleichheit der Placentahälften wurde durch Bildung einer Placenta marginata nach der benachtheiligten Seite hin und durch Bestehenbleiben von zwei Anastomosen zwischen den beiden Placentakreisläufen (Placentatypus D) ausgeglichen. Trotz der ursprünglich so ungleichen Lage beider Allantoiden kam es also nicht zur Acardie, sondern zu gleichmässiger Entwickelung beider Zwillinge.

Aehnliche Fälle, wenn auch nicht von so starker ursprünglicher asymmetrischer Anlage beider Allantoiden und doch gleichmässiger Entwickelung der Zwillinge haben veröffentlicht Cornelius (Ceber die Einpflanzung der Nabelschnur in die Eihäute. Diss. Marburg. 1859. S. 35) und C. Ruge (Beiträge zur Geburtsh. und Gynäłol. Berlin. I. Sitzungsberichte. S. 45).

Die Hypothese von Ah]feld, dass der Acardius ein Allantoisparasit sei, ist übrigens, wenn auch weniger prononcirt, schon von Claudius ausgesprochen worden.

Claudius unterscheidet 4 Arten, wie die beiden Allantoiden eines Zwillingseies sich gegeneinander verhalten: A. a. 0. S. 25. „1. Setzen sich bei einer Zwillingsschwangerschaft die Allantoiden der beiden Eier (Claudius hat noch die Meinung, dass zwei Eier dureh Verschmelzung der beiden Chorien und Resorption der Zwischenwand sich wirklich vereinigen können) in gehöriger Entfernung von einander an und schicken ihre Aeste nach entgegengesetzten Richtungen, so entstehteine conglutinirte Doppelplacenta mit einander nahe gerückten Nabelsträngen; 2. verlaufen die mittleren und kleineren Aeste gegen einander, so entsteht die scheinbar einfache mit vielfachen Anastomosen, wobei die Chorien zu einem einfachen verschmelzen; 3. treffen aber die Hauptarterien auf einander, so anastomosiren sie und die Acardiacusplacenta bildet sich. Diese ist also anfangs auch doppelt; indem aber bald nach der Vereinigung alles Blut nur aus den Arterien eines Nabelstranges kommt, strecken sich bei dem Wachsthum der Placenta auch die Aeste des anderen in der Richtung des eintretenden Blutstromes, sodass sie bei vollendeter Ausbildung nicht von den Aesten der ursprünglich jenem angebörigen Arterien und Venen unterschieden werden können......; 4. Es giebt noch eine vierte Form. Trifft nämlich durch eine Verirrung die Allantois des einen auf den Nabelstrang des anderen, so kommt es gar nicht zur Placentarbildung auf Seite des später kommenden Foetus, sondern es entstehen die gegabelten Nabelstränge und die Folge ist ebenfalls die Bildung eines herzlosen Foetus. Hierher gehört die von Hempel abgebildete Placenta, wo beide Nabelstränge sich an der Insertionsstelle auf der Placenta verbinden."

No. 4 stellt den "Allontoisparasit" vor. 
Mit Weglassung der uncorrecten Vorstellung von Claudius von der Vereinigung zweier Eier hat Ahlfeld die Hypothese in folgender Weise ausgedrückt:

Der Einfachheit halber setze ich der Hypothese von Ahlfeld meine Kritik derselben gleich gegenüber.

\section{Hypothese von Ahlfeld.}

1. „Die Entstehung der Acardiaci ist auf eine sehr frühe Zeit zurückzuverlegen, nämlich auf die Zeit gleich nach dem Hervorsprossen der Allantois.

Vielleicht giebt es Acardiaci, bei denen bereits ein Wechsel der Strömung des Blutes in den Dottergefässen vor sich ging.
2. Am deutlichsten kann man sich die Entstehung vorstellen, wenn man annimmt, dass die cine Allantois die Innenfläche des Eies bereits vollständig umwachsen hat, während die andere Allantois eben erst sich ausbreitet. So muss nothwendiger Weise die zweite Allantois durch das Gewebe der ersten hindurchwachsen, will sie zur Decidua vera gelangen.

\section{Kritik.}

ad 1. Allerdings ontstehen Acardii auch schon vor Hervorsprossen der Allantois auf dem Wege der Dottergefässcirculation (wenn die Dotter gemeinschaftlich ist). Solche Acardii sind aber stets Epiphyten, welche keine eigene Nabelschnur haben und deshalb hier nicht besprochen werden.

Freie, d. i. mit Nabelschnur versehene Acardii können nicht nur entstehen gleich nach Hervorsprossen oder besser nach der Vereinigung der beiden Allantoiden, sondern auch sehr spät, d. i. bis zur Mitte der Schwangerschaft, wie ich unten beweisen werde. Ablfeld's Zeitangabe ist also viel $\mathrm{zu}$ beschränkt.

ad 2. Man kann das Zuspätkommen der zweiten Allantois recht wohl als möglich wenn auch nicht als wahrscheinlich - zugeben. Trotzdem folgt doch daraus nicht, was Ahlfeld folgert. Denn 
3. Dann öffnet sich nicht ein Gefäss des einen Fötus in ein Gefäss des anderen, wie es Claudius beschreibt, sondern eine Unmasse kleiner Allantoisgefässe tritt mit den Allantoisgefässen der anderen Frucht zusammen.

Der Blutkreislauf des stärkeren Herzens, identisch mit dem Blutkreislaufe der Frucht, deren Allantois zuerst sich entwickelte, überwindet den Strom in den Capillaren und gelangt so in den Körper der zweiten Frucht." ad 3. Unter allen Umständen - mögen beide Allantoiden gleichzeitig oder nach einander nach der Innenffäche der serösen Hülle hin und ihr aufwachsen -verwachsen sie dadurch, dass ihre Gefässverbindungen unzählige werden, zu einem einzigen primären Choriongefässnetz, welches functionell beiden Zwillingen durchaus gemeinschaftlich gehört, selbst wenn der eine ursprünglich nur einen kleinen Theil desselben gebildet haben oder gar nur auf die Innenfläche der Ausbreitung der ersten Allantois aufgetroffen sein sollte.

Bezüglich der allseitigen Verbindung der beiderseitigen Allantoisgefässe hat Ahlfeld eine richtige Vorstellung. Er zieht aber daraus nicht die richtigen Schlüsse. Die unzähligen und im Anfang durchweg capillaren Verbindungen zwischen beiden Allantoiden sind entweder arteriell-arteriell oder arteriellvenös oder venös-venös. Ahlfeld nimmt stillschweigend an, dass der Zwilling, dessen Allantois zu spät kommt, geringeren arteriellen Blutdruck habe, als derjenige, dessen Allantois schon auf der serösen Hülle ausgebreitet ist. Diese Annahme ist willkürlich. Die Wachsthumsgrösse und der arterielle Druck brauchen nicht parallel zu gehen, thun es oft nicht. Ausserdem kann die später kommende Allantois auch 
lediglįch wregen des etwa grösseren Weges später kommen. Wir wollen aber die Annahme von Ahlfeld einmal gelten lassen. Dann wird allerdings durch die arteriell-arteriellen Verbindungen Blut vom träftigeren Zwilling zum schwächeren fliessen. Dort aber wird es nur so lange dem arteriellen Blutstrom des schwächeren Herzen entgegenfliessen, als das Gefässlumen, welches sich ihm im fremden Gefässnetz zur Verfügung stellt, nicht wesentlich grösser ist, als dasjenige der Anastomosen. Sobald es in deutlich weitere Arterien des fremden Gefässnetzes gelangt, wird sein Druck schnell viel geringer, und es fliesst in der Richtung des Blutstroms des schwächeren Zwillings weiter und mit demselben in das Venensystem des schwächeren. Die entgegengesetzte Richtung des überfliessenden Blutes, d. i. Aufstaung nach dem schwächeren Herzen hin wäre nur möglich, wenn ein Ausweichen nach der Seite der Venen hin nicht möglich wäre. Ist das vom stärkeren Zwilling ber übergeflossene Blut in den Venen des schwächeren angekommen, so fliesst es mit dessen Blut dem schwächeren Herzen in normaler Richtung zu, und regt dieses durch stärkere Füllung und bessere Ernährung zu grösserer Action an. Das ursprünglich schwächere Herz wird also gekräftigt, und zwar 
so lange, bis es durch seinen wachsenden Blutdruck das weitere Ueberströmen des fremden Blutes selbst verhindert.

Ein Gleiches wird das Blut thun, welches etwa durch die venösvenösen Verbindungen vom kräftigeren Zwilling zum schwächeren gelangt, und ebenso dasjenige, welches von den Arterien des kräftigeren Zwillings durch die arteriell-venösen Verbindungen direct in die Venen des schwächeren mehr gelangt, als umgekehrt von den Arterien des schwächeren in die Venen des stärkeren. Durch die ungleiche gegenseitige Transfusion, welche durch stärkeren Blutdruck auf Seiten des einen Zwillings zu Stande kommt, wird also immer der schwächere Zwilling stärker, der stärkere schwächer werden. Es wird durch sie immer Gleichheit, oder eine gewisse Ausgleichung, nicht aber Unterliegen des schwachen Herzens eintreten.

4. Später sagt Ahlfeld, ad 4. Damit wird aber die um die Vorstellung der Umkehr zu erleichtern (Berichte und Arbeiten 1881-82, S. 273), er habe die Umkehr der Blutströme in den Nabelschnurgefässen in eine Zeit verlegt, in welcher das Blut noch nicht in geschlossenen Röhren kreist, sondern in den Bindegewebslücken des Allantoisstratums sich einen Weg bahnt. Vorstellung noch mehr unphysikalisch. Wie soll Blut, das nicht in geschlossenen Röhren kreist; überhaupt seinen Weg zum Herzen zurückfinden? 
5. "Ist die Allantois der zweiten Frucht in allen ihren Theilen mit der Allantois der ersten Frucht vereinigt und participirt sie von Anfang an in keiner Weise an den Chorionzotten des Placentatheiles, so entsteht der reine Allantoisparasit, die Nabelschnurgefässe setzen sich velamentös in die Placenta ein und gehen direct zur Insertion der Nabelschnur des Autositen (?)

Hat sich nur ein Theil der Allantois mit der Allantois der ersten Frucht verbunden, während ein anderer Theil direct zu den Chorionzotten und zur Decidua gelangen konnte, so wird erst nach und nach der Parasit vom Autositen in Beschlag genommen. Dann kann eine Zeit lang ein doppelter Blutkreislauf im Parasiten vorhanden sein, dann kann das Herz, wenn auch rudimentär, persistiren und bis zu einem gewissen Zeitpunkte thätig sein, dann inserirt in der Regel die Nabelschnur marginal oder auch, wenn das Herz gut ausgebildet ist, auf der Placenta".

Deutlicher spricht Ahlfeld seine Vorstellung aus in: Die Missbildungen des Menschen Seite 45: „Die Nabelschnur des Acardiacus inserirt in der Regel velamentös, je weiter aber das Herz des Acardiacus ausgebildet ist, um so eher inserịt sein Nabelstrang neben dem ad 5. Dieses Schema wäre sehr bequem, wenn es nur auch von der Natur befolgt würde: Zuerst trifft es in Wirklichkeit gar nicht zn, dass das Herz des Hemiacardius um so vollkommener ausgebildet angetroffen werden soll, je näher die Nabelschnur desselben nach der Placenta resp. nach deren Mitte hin inserirt ist. Es existirt da gar kein Parallelismus. Z. B. fehlt dem Falle von Breus, wo die Nabelschnur des Acardius in der Mitte der Placenta auftrifft, jedes Herz und dabei ist der Acardius sehr vollkommen ausgebildet. Andere Male sind wieder bei ebensolcher gemeinschaftlichen centralen Insertion der Nabelschnur die Herzen beider Zwillinge durchaus gleich vollkommen ausgebildet. Nicht ganz selten finden wir umgekehrt die Nabelschnur des zweiten Zwillings gerade gegenüber der Placenta in den Häuten inserirt und doch die Herzen beider gleich gut ausgebildet. Dann sieht man aber auch gar keinen Grund, warum, wenn die erste Allantois die ganze seröse Hülle besetzt bat, die zweite Allantois nicht doch auf die Gegegend der Eiinsertion auftreffen soll, so dass die Nabelschnur also später auch auf der Placenta inserirt, ohne dass damit das Herz länger bestanden zu haben - später abgestorben zu 
der gesunden Frucht im Centrum der Placenta. sein - brancht als in anderen Fällen.

Weiter nimmt Ahlfeld an, dass die 2. Allantois theilweise auf die erste Allantois treffen und sich mit dieser verbinden, zugleich aber theilweise auf die unbesetzte Stelle treffen kann. Es ist dies nur dann denkbar, wenn die 2. Allantois gerade auf den Rand der eben vorhandenen. Ausbreitung der ersten Allantois treffen würde. Denn sobald und wo Allantois auf Allantois eigene oder fremde - trifft, tritt Verwachsung ein und hört alsbald weitere Ausbreitung auf, etwa wie bei Epithelüberwachsung bei Wunden der Haut. Träfe also die 2. Allantois auf die Ausbreitung der ersten - aber nicht auf ihren Rand - so würde einfach Verwachsung, aber nicht ein Weiterwuchern der zweiten Allantois eintreten und die noch nicht besetzte Stelle der serösen Hülle würde weiter von der ersten Allantois überwachsen werden.

Ahlfeld scheint die Vorstellung zu haben, dass je grösser die Allantoisausbreitung ist, um so besser auch die Ernährung und Herzkraft des zugehörigen Zwillings, so dass dessen Herzkraft danu die des Mitzwillings um so eher überwindet. Beides ist nur unter ganz bestimmten Bedingungen des Gefässarrangements, durchaus aber nicht stets der Fall. 
6. Es giebt eine vierte Form des Acardiacus, die ich mit dem Namen Acardiacus anceps bezeichne, bei der das Herz regelmässig, wenn auch rudimentärvorhanden ist, eine Zeit lang, in einigen Fällen vielleicht bis zum Ende der Schwangerschaft thätig ist.

7. Die Ausbildung des Körpers des Acardiacus hängt weniger von dem Orte $a b$, wo die Nabelschnur mündet, sondern von der Zeit der Entstehung, von der Quantität, nicht aber von der Qualität des zugeführten Blutes, vom Grade der Abhängigkeit dem Autositen gegenüber und dementsprechend von der Ausbildung des eigenen Herzens. Am weitesten ist die Körperform beim Acardiacus anceps entwickelt. Es pflegen bei diesex Unterart Kopf, Rumpf, Becken und Extremitäten gleichmässig entwickelt zu sein, während in den übrigen Unterarten immer nur der Kopf mit dem Rumpfe oder nur das Becken mit dem Rumpfe sich ausbilden.
Unter bestimmten Bedingungen der Gefässanordnung kann sogar das Gegentheil der Fall sein.

ad 6. Man sieht keinen Grund die unter 5 rubricirten Fälle mit rudimentären Herzen von denjenigen unter 6 zu trennen. ad 7. Allerdings sind die Hemiacardii (Acardiaci ancipites nach Ahlfeld) im Allgemeinen am besten entwickelt, weil bei ihnen die Blutcirculation von zwei Herzen und deshalb wenigstens nicht leicht ganz unvollständig besorgt wird.

Doch kann die Körperausbildung des Acardius auch die denkbar rollkommenste sein, ohne dass ein eigenes Herz existirt (Fall von Breus), weil auch hier die Blutversorgung eine recht gute sein kann.

Die Verhältnisse liegen durchaus micht so einfach, wie os nah $A$ hlfeld sein müsste. Das wirkliche Verhältniss zu zeigen, wird jedoch erst in meiner nunmehr folgenden ausführlichen Arbeit möglich sein. In dieser wird auch, und zwar besonders in Gruppe VII, durch Gegenüberstellung der Fälle von de la Faille und Ratbke deutlich zu Tage treten, wie die ganze Hypothese Ahlfeld's rom Allantois- 
parasiten nicht zu brauchen ist. Denn obgleich in diesen beiden Fällen die Allantois des 2. Zwillings bez, 3. und 4. Vierlings im Kopfe des ersten inserirt, also von diesem so abhängig ist, wie nur möglich, so ist doch nur in dem Falle von de la Faille, nicht aber in dem Falle von Rathke Acardie eingetrten.

\section{Figene Untersuchungen.}

Ich hatte zuerst die Absicht, dem Leser das ganze in der Literatur vorhandene, die Acardii betreffende Beobachtungsmaterial systematisch geordnet vorzuführen und, indem ich aus demselben die nöthigen Schlüsse zöge, eine auf breitester Basis gegründete Hypothese aufzubauen.

Ich habe von dieser Absicht aber bald abgehen müssen. Es würde dabei nicht nur der. Leser gar zu bald in dem Wust unvollkommener Beobachtungen ermüdet werden, sondern es würde der grössere Theil der Beobachtungen nicht einmal in ein der Aetiologie entsprechendes System eingefügt werden können.

Bei vielen Beobachtungen ist dies nicht möglich, weil sie nicht genau genug beschrieben sind; bei noch mehr, weil an der sonst guten Beschreibung einzelne Theile, z. B. die (meist nicht zur Verfügung gewesene) Placenta, ganz fehlen; bei den meisten aber, weil zur Zeit der Geburt an dem ausgebildeten Acardius und an dessen Placenta, wenn sie auch noch so gut beschrieben sind, die Art der Entstehung des Acardins nicht mehr oder nicht mehr mit derjenigen Sicherheit zu erkennen ist, dass aus ihrer vollständigen Aufzählung eine grössere Beweiskraft resultirte.

Ich muss mich also darauf beschränken von dem Material der Literatur nur einzelne und genügend beschriebene Fälle, sei es als Beweis, sei es als Beispiel vorzuführen.

Aber auch bei solcher Auswahl der Fälle würde die Darstellung immer noch schwerfällig und wenig durchsichtig werden, wenn die Fälle in dieser selbst Platz fänden. Manche Fälle bedürfen einer eingehenden Besprechung, weil sie überhaupt recht complicirt sind, andere weil sie von den Autoren in ganz anderem Sinne verstanden und verwerthet worden sind, als es von mir geschehen wird. Ich ziehe also vor, die Resultate meiner Untersuchungen zuerst zusammenhängend und ohne casuistische und literarische Episoden vorzutragen und erst nachher die einzelnen zu beweisen- 
den oder zu besprechenden Punkte mit der nöthigen Casuistik in Form von kleinen Sonderarbeiten folgen zu lassen.

Dadurch werden zwar Wiederholungen unvermeidlich. Dieselben werden aber das an sich ziemlich schwierige Verständniss nur erleichtern. Es bleibt dann dem Leser, der sich in der ersten allgemeinen Darstellung orientirt hat, freigestellt, in den Sonderdarstellungen für jede ihn besonders interessirende Frage genauere Darlegung mit dem beweisenden Material aufzusuchen.

\section{Umgrenzung des Begriffes. - Nomenclatur. -}

Die Namen, welche den Acardiis und ihren Verwandten bisher gegeben worden sind, entsprechen, weil sie sich fast nur auf künstliche Systeme stützen, fast durchweg nicht den Ansprüchen, welche ein natürliches System an sie stellen muss.

Den von Ahlfeld gebrauchten Namen "Allantoisparasit" habe ich schon zurïckgewiesen. Der Ausdruck „Parasit" muss nach dem bisherigem allgemeinen Gebrauch für solche Individuen reservirt bleiben, welche ihre Nahrung dauernd von einem anderen Individuum beziehen. Nun scheinen allerdings eine Anzahl Acardii diesem Erfordernisse ganz zu entsprechen. Aber ein grosser Theil hat wirklich seine eigene Placenta noch, und fast alle haben eine solche gehabt. Da man nun die in dieser Richtung ganz regelrecht fortlaufende Reihe nicht wird unterbrechen wollen, so wird man den Namen „Parasit" für die freien Acardii gar nicht brauchen dürfen. Er wird in der Teratologie für diejenigen Individuen eines Doppelmonstrums verbleiben müssen, welches keinen eigenen Nabelstrang hat.

Den Namen "Allantoisparasit" könnte ich nur für die Fälle als berechtigt anerkennen, wo die Allantois und später die Nabelschnur sich an einem Körpertheil des anderen Zwillings inserirt und von dort alle Nahrung bezieht (s. unten Gruppe ViII).

Der Name "Omphalosit" von Is. Saint Hilaire kann ebenfalls nicht bei Bestand bleiben, wenn ihn auch Dareste angenommen hat, denn er schliesst die falsche Vorstellung von SaintHilaire in sich, dass die Placentagefässe des Fötus mit denen der Mutter communiciren, "Monstres omphalosites, ou vivants seulement d'une vie imparfaite, et pour ainsi dire passive, qui n'est entretenue, que par la communication avec la mère, et cesse dès que le cordon est rompu."

Der von Daresto dafür vorgeschlagene Namen "Adelpho- 
site" ist etwas richtiger, aber überflüssig, demn jeder eineiige Zwilling ist seines Mitzwillings Adelphosite.

Taruffi nennt die Acardii Disomata omphalo-angiopaga (disomi omfalo-angiopaghi), welche mit den Disomata monochoria (disomi monocorii) zusammen die Abtheilung Disomata dierita (disomi diereti) bilden.

Ballantyne (Studies in foetal Pathologie and Teratolgy, Transact. of the Obstetr. Soc. of Edinburgh, 12. December 1892) sagt für Omphalo-angiopagi "Allantoido-angiopagi“, um ein Missverständniss durch die Dottergefässe zu vermeiden, welche die Engländer "Omphalo-mesenteric vessels" nennen.

Aber in Wirklichkeit sind alle Disomata monochoria auch Disomata ophalo-angiospaga und umgekehrt, und nur durch ein Uebereinkommen könnte man den ersten Namen für die getrennten normalen eineiigen Zwillinge und den zweiten für diejenigen getrennten eineiigen Zwillinge brauchen, von denen einer missgestaltet ist. An sich bedeuten diese Namen das nicht. Ja unter „Sternoetc. pagi" versteht man sogar nur vereinigte normale Zwillinge. Solche Termini technici sind bedenklich, mindestens dann, wenn man bessere haben kann.

Auch der Vorschlag von Hirschbruch (Dissert., Berlin 1895) jeden Acardius "Gemellus placento-parasiticus" zu nennen, ist nicht annehmbar. Denn 1. ist der Acardius von vornherein gewöhnlich gar nicht Placentaparasit, sondern er hat seine eigene Placenta, die ihm erst secundär weggenommen wird. Einen so beraubten wird man kaum "Parasit" nennen wollen; im jetzigen Sprachgebrauch ist ja der Parasit der Räuber. 2. besitzen die Uebergangsformen in Wirklichkeit ihre Placenta mehr weniger vollkommen noch. Es wäre unmöglich, eine sichere Grenze zu finden. Mindestens wäre die fortlaufende Reihe ohne Noth und gegen die Zweckmässigkeit getrennt.

Das natürliche System, welches sich aus den Resultaten meiner Untersuchungen von selbst ergiebt, thut am besten die (allein hier zu behandelnden) getrennten eineiigen Zwillinge, welche stets Gefässverbindungen in der Placenta haben, 1. nach der Art dieser Verbindungen und 2. nach der Wirkung derselben zu ordnen.

Da die Zottengefässverbindungen bei allen vorhanden sind, so brauchen sie niemals besonders genannt zu werden. Es sind also nur die Verbindungen durch die (oberflächlichen) Anastomosen zu nennen - also ob ohne resp. mit Anastomosen.

Da die Wirkungen der Gefässverbindungen sich am Empfind- 
lichsten am Herzen der Zwillinge zeigen, so wird man die Unterabtheilungen am Besten nach dem Herzen benennen. Man kommt also auf die ältere Bezeichnung nAcardius" zurück. Nan muss sie aber durch Zusätze und Modificationen gut formbar machen und die so gewonnenen einzelnen Termini technici scharf begrenzen.

Von den beiden Bezeichnungen -- Acardiacus - Acardius hat die letztere, welche in neuerer Zeit wiederholt gebraucht worden sind, vor der ersteren und älteren medicinisch bez. naturwissenschaftlich keinen, aber auch philologisch, wie mir mehrere Philologen versichern, keinen solchen Vorzug, dass man berechtigt wäre, deshalb allein von der ersten, früher allgemein gebrauchten Bezeichnung abzugehen Dennoch sehe ich mich dazu veranlasst, um die daraus neuzubildenden Namen möglichst kurz zu fassen z. B. statt Hemiacardiacus zu sagen Hemiacardius, statt Pseudohemiacardiacus Pseudohemiacardius etc.

Ohne ohne scharfe Umgrenzung dessen, was man unter dem Begriff "Acardius" "Acardie" zusammenfassen soll, können grobe Missverständnisse nicht vermieden werden.

Allgemein anerkannt ist bereits, dass der Name Acardius nicht ganz wörtlich zu nehmen ist und dass ein Acardius recht wohl ein (mehr weniger rudimentäres) Herz besitzen kann, ja dass dieses sogar bis zur Geburt in Function gewesen sein kann.

Welches ist nun das ausschlaggebende Merkmal, das den Acardius von einem anderen Fötus unterscheidet; wenn es nicht die An- oder Abwesenheit eines Herzens ist?

Eine bestimmte mangelhafte Beschaffenheit des Herzens kann auch nicht die Grenze bilden; denn wir haben Einlinge, - - also ganz selbstständige Embryonen — deren Herz nicht vollkommener gebaut ist, als das mancher Acardii, die als solche allgemein anerkannt sind.

Noch weniger kann die Beschaffenheit des übrigen Körpers massgebend sein. Wir finden einerseits Acardii, welche sich abgesehen vom Mangel des Herzens - bezüglich der Vollzähligkeit der Organe von einer gewöhnlichen Frucht so gut wie nicht unterscheiden, z. B. der Fall von Breus (Wiener med. Jahrb. 1884. S. $57 \mathrm{ff}$ ). Andererseits giebt es Einlinge, welche ja gar nicht Acardii sein können, welche doch am Körper die gleichen Defecte zeigen, wie sie an einem deutlichen Acardius vorkommen.

Wissenschaftlich haltbar ist nur diejenige Erklärung des Begriffes Acardie, welche sich anf die Blutcirculation im betreffenden 
Foetus stütat, also nicht auf das anatomische Vorhandensein eines Herzens, sondern auf die physiologische Thätigkeit eines solchen.

Acardie ist also dann vorhanden, wenn in einem Zwillingsembryo die Blutcirculation ganz oder wenigstens theilweise vom fremden Herzen besorgt wird, und zwar (in einem Theil des Gefässsystems) in der der Norm entgegengesetzten Rịchtung ${ }^{1}$ ).

1) Ahlfeld begeht den Fehler, dieses Merkmal nicht einzuhalten; und es muss daraus unlösbare Verwirrung entstehen.

Von den 9 Fällen, welche AhIfeld als Acardii mit Herz aufführt (Einladungsschrift zur Feier des 25jährigen Bestehens der Gesellschaft t. Geburtshülfe zu Leipzig) sind mehrere Fälle durchaus nicht Acardii, auch nicht in dem Sinne, wie sie Ahlfeld auffasst: Acardii ancipites (Hemiacardii).

Der Fall Betschler-Tamm (De hydrope foetus anasarca, Dissert., Breslau 1857) hatte, wie ich es früher (Dieses Arch., Bd. XXIX, S. 426-429) schon darlegte, mvollständigen Situs transversus und damit verschiedene Defecte. Er hatte aber ein normales Herz, und es ist gar nicht nachzuweisen, dass das Blut in irgend einem 'Theile seiner Gefässe oder in einem Nabelschnurgefäss in abnormer Richtung sich bewegt hätte.

Ebenso ist der von Ahlfeld mit als Acardius anceps aufgeführte und auf den ersten Blick allerdings einem Hemiacardius sehr ähnliche Fall von Barkow (Beitr. zur patholog. Entwicklungsgeschichte, II., S. 2 ff.) gar kein Hemiacardius, sondern auch er hat nur partiellen Situs transversus mit reichlichen Defecten. Das Herz hat nur eine Torkammer und eine Kammer. Aber die Blutcirculation kann nach der Beschaffenheit der Gefässe nur in normaler Weise vor sich gegangen sein. Barkow erkennt dies auch sehr wohl und sagt, indem er damit den Acardius von Otto (Museum anatom.-pathol., Vratisl. 1851, Fol. P. 154, 155, N. CCLXI, T. IX, Fig. 1) vergleicht und im Uebrigen sehr ähnlich findet:

„In Beziehung auf die Ausbildung des Gefässsystems zeigte sich demnach in dem von 0 tto und in dem von mir beschriebenen $F$ all eine wesentliche Versehiedenheit. In jenem fehlte das Herz, in diesem war es vorhanden. Die Gegenwart desselben, selbst in seiner einfachen Ausbildung, lässt darüber keinen Zweifel, dass der von mir untersuchte Pseudathorax seinen selbstständigen Blutlauf hatte, dass er, auch wenn seine Placenta mit den Placenten seiner Drillingsgeschwister in Verbindung gestanden haben mag, und wenn auch Anastomosen ihrer Placentagefässe stattgefunden haben sollten, doch nicht ein blosser Parasit seiner Geschwister gewesen, sondern durch seine eigenen Placentagefässe in Beziehung zu den Uteringefässen gestanden hat. Durch die Vena umbilicalis, welche in ihrem Verlauf in der Unterleibshöhle an der Stelle der Art. umbil. dextra an der rechten Seite der Harnblase verlief, gelangte das Blut von der Placenta zur Vena cava, aus dieser in die Herzvorkammer, sodann in die Herzkammer, durch die Aorta zu den verschiedenen Theilen des Körpers und durch die Arteria umbilicalis zur Placenta zurücls," 
Wird die Circulation im Acardius ganz vom fremden Herzen besorgt, so wird die Acardie als vollkommen (Holoacardie) angesehen, gleichgültig, ob sich dabei anatomisch ein mehr weniger vollkommenes Herz vorfindet oder nicht.

Wird die Circulation im Acardius nur theilweise rom fremden Herzen besorgt, so nenne ich den Zustand Hemiacardie. Bei dieser wird natürlich die Grenze der beiderseitigen Kreisläufe (Circulationsäquator) den Fötus nicht gerade in zwei ganz gleiche Hälften zu theilen brauchen. Auch dieser Name ist also nicht streng zu verstehen. Es genügt zur Hemiacardie schon einerseits, wenn das eigene Herz nur einen sehr kleinen Theil des Körpers mit Blut versorgt, während der viel grössere vom fremden Herzen versorgt wird, und es genügt zur Hemiacardie andererseits auch schon, wenn das Blut nur in einer Nabelschnurarterie in umgekehrter Richtung läuft, und wenn dadurch etwa nur eine untere Extremität oder noch weniger vom fremden Herzen her mit Blut versorgt wird.

In Wahrheit lässt sich denn auch in dem gut beschriebenen und abgebildeten Präparat gar kein Grund finden, warum das Herz nicht sollte solchen Kreislauf unterhalten haben.

Der Fall ist also kein Acardius. Freilich ist er deshalb nicht weniger, sondern noch mehr interessant. Er stellt, wie wir unten in Gruppe III sehen werden, ätiologisch einen misslungenen Acardius dar, der allerdings unbedingt ein Acardius geworden wäre, wenn dazu nicht die Anastomosen auf der Placenta gefehlt hätten. Er ist ein Pseudohemiacardius.

Ahlfeld hat auch sonst noch einer Reihe von Fällen Gewalt angethan, um sie für seine Hypothese brauchbar zu machen. Wenn aber Köhler, Wernher, Brodie und Poppel ganz bestimmt anssagen, dass in ihren Fällen ein Herz nicht vorhanden war, so geht es unmöglich an, das zu bezweifeln, wie es Ahlfeld thut (Archiv, XIV., S. 357). Im Gegentheil! Wenn sich so viele Fälle mit der Hypothese nicht vereinigen lassen, so wird wohl eher diese unrichtig sein', als die Aussage einer ganzen Reihe guter Beobachter.

Dem Fehler, dass ein vorhandenes, aber schon längst abgestorbenes, d. h. nicht mehr thätiges Herz, noch gewissermaassen als voll betrachtet wird, begegnet man in der Literatur noch recht häufig. Z. B. Der Acephalus abrachius von Slymann (Transactions of the obstetr. Soc. of London, XXXI., 1889, S. 258 ff.) wird ein Acardiacus anceps oder paracephalus genannt, weil er ein rudimentäres Herz hatte. Dieses Herz hatte aber schon lange Zeit nicht mehr gearbeitet. Der Zusammenhang mit der Aorta war obliterirt. Ob solches Herz im Acardius vollständig schwindet oder durch Ernährung aus der Umgebung noch mehr weniger lange - hier bis zur Geburt im VI. Monat -- vor gänzlichem Zerfall geschützt und damit anatomisch, aber nicht physiologisch erhalten wird, darauf kann es nicht ankommen. 
Zwischen diesen beiden Extremen der ungleichmässigen Zweitheilung des Hemiacardius durch den Circulationsäquator liegen alle möglichen Variationen minder ungleichmässiger Theilung und so ist in den Hemiacardiis ein sehr breiter und gleichmässiger Uebergang von den sonst so isolirt stehenden Holoacardiis (gewöhnlich einfach Acardii genannt) zu den normalen Früchten geschaffen - eine fortlaufende Reihe von der grössten Abnormität in dieser Richtung bis zum Normalen.

Diese Reihe umfasst aber eine Anzahl von Missbildungen nicht, welche den. Acardiis sowohl nach Form als auch nach Aetiologie analog sind. Sie bilden für sich allein wieder eine besondere, der Reihe der Acardii ganz analoge Reihe, unterscheiden sich aber von den Acardiis dadurch, dass bei ihnen die Circulation nirgends umgekehrt ist, und dadurch, dass auf der Placenta die beiden Anastomosen, welche bei den Acardiis stets vorhanden sind (arterielle und venöse Anastomose) ebenso regelmässig fehlen. Ich nenne sie Pseudoacardii.

Wie die Acardie als Gesammtbegriff die Holoacardie und Hemiacardie umfasst, so müsste auch die Pseudoacardie als Gesammtbegriff die Pseudoholoacardie und die Pseudohemiacardie umfassen.

Da aber die Pseudoholoacardii, wie wir sehen werden, einfach todte Embryonen sind, und da also in der Reihe der Pseudoacardie nur die Pseudohemiacardii übrig bleiben, so werden die Ausdrücke Pseudohemiacardii und Pseudoacardii sachlich synonym. -

Die Bezeichnungen Microcardie und Macrocardie für die Fälle, deren relatives Herzgewicht (bei normaler Form des Herzens) die Zone des normalen Herzgewichtes nach unten bez. nach oben überschreitet, sind für sich verständlich. -

Ich werde in Folgenden wiederholt von Herztod und weiter von primärem und secundärem Herztod sprechen müssen.

Diese Ausdrücke sind, so wie ich sie brauche, nicht ohne Weiteres verständlich und ich muss, um Missverständnisse zu vermeiden, eine Erklärung vorausschicken.

Herztod. Wenn ein eineiiger Zwilling zum Holoacardius werden soll, so gehört dazu zwar, dass sein Herz aufhört zu zu arbeiten, d. h. abstirbt. Der Zwilling darf dabei aber nicht auch in allen seinen übrigen Organen mit absterben. Abgestorbene Organe können nicht wieder aufleben und würden sich auch nicht wieder ersetzen. Die später am Acardius noch vorhandenen und noch lebenden, weil wachsenden Organe können also nicht abge- 
storben gewesen sein. Wir müssen demnach beim Acardius Tod des Herzens und Tod anderer Organe bez. des ganzen übrigen Körpers unterscheiden. Es ist zwar nicht unwahrscheinlich, dass überhaupt der Tod eines Embryo immer zuerst nur Herztod ist. Man könnte also den Ausdruck Herztod allenfalls auch für gewöhnliche abgestorbene Früchte brauchen. Es hätte dies aber keinen Nutzen, weil bei einem gewöhnlichen Embryo nach dem Herztod schnell auch der ganze übrige Körper abstirbt. Beim Acardius jedoch müssen Herztod und Gesammttod streng unterschieden werden und man darf bei einem Zwilling, welcher zum Acardius wird, nicht einfach ron Tod, sondern zunächst nur von Herztod sprechen.

Dieser Herztod kann, wie unten genauer gezeigt werden wird, auf verschiedene Weise zu Stande kommen. Für die folgenden Untersuchungen ist es nur nöthig, zwei Hauptarten von Herztod zu unterscheiden.

Bei der einen Art stirbt das Herz ab in Folge von anatomischen Störungen im Blutgefässsystem, indem - zumeist durch absolute oder nur relative Verengerungen etc. an bestimmten Stellen des Gefässsystems - dem Herzen die für seine zweckmässige Arbeit und für seine Ernährung nöthige Blutzufuhr -- Speisung beschränkt oder ganz abgeschnitten wird. Gewöhnlich tritt durch die fortsehreitende Abnahme der Speisung zunächst nur immer wachsende Schwächung (und Verkleinerung) des Herzens ein - erst dann wird das zu stark geschwächte Herz durch den für dasselbe zu hohen Druck des andrängenden fremden Blutes zum Stillstand gebracht - getödtet.

Je nach dem Ort und der Art der primären Störung im Gefässsystem werde ich diese Todesart wieder in Unterabtheilungen zerlegen.

Stellt das Herz seine Thätigkeit aus einer anderen Ursache als in Folge von Störungen im Gefässsystem ein, so nenne ich dies primären Herztod. Wirklich primär ist natürlich auch dieser Herztod kaum je. Es werden auch bei ihm fast stets wieder Ursachen vorhanden sein, wie wir solche in ihrer Mannigfaltigkeit zu beobachten in der Klinik genug Gelegenheit haben: überbohe Temperatur, Mangel an Sauerstoff ete. etc. Das "primär" bedeutet hier also nur nohne Schuld des Gefässsystems", und ist nur der Gegensatz gegen jenes "secundär".

Genau genommen würde mein "primärer Herztod" je nach 
seiner verschiedenen Ursache ebenfalls in Unterabtheilungen zerfallen müssen. Doch besitze ich noch nicht genügend viele und genügend sicher untersuchte Fälle von Acardiis mit solcher Todesart, um auch da schon genau präcisiren und unterscheiden zu können. Ich muss mich also begnügen, bei dieser Entstehung der Acardie überhaupt erst Herztod ohne ursächliche Betheiligung des Gefässsystems constatiren zu können. Einen Nachtheil hat dies nicht. Denn die Unterscheidung von verschiedenen Arten primären Herztodes hätte für die Erklärung der Entstehung der Acardie keinen weiteren Nutzen.

\section{Einschränkung des Themas.}

Es giebt Acardii aus drei ganz verschiedenen Entwickelungsperioden des Embryo.

1. Acardii, bei welchen sich ein Herz überhaupt nicht entwickelt hat.

Die ersten Gefässe des Embryo entstehen nach den Untersuchungen von His gar nicht im Embryo, sondern von den Gefässen der Area vasculosa her als Sprossen, welche in den Körper des Embryo eindringen. Das Herz entsteht allerdings, was seine Muskelschicht betrifft, im Embryo selbst, erhält aber seine Endothelschläuche auch erst von den heranwachsenden Gefässen.

Es braucht dieser Vorgang bei einem eineiigen Zwilling nur gehemmt zu werden — und Missbildungen sind ja zumeist nur Hemmungsbildungen -; es braucht dann weiter nur die Area vasculosa beider Zwillinge mit einander so vereinigt oder einander so nahe zu sein, dass die sich bildenden Gefässe beider genügend reichlich anastomosiren, so wird aus jenem Zwillinge in dem Sinne der Meckel-Dareste-Panum'schen Hypothese ein Acardius werden, weil sein Herz gar nicht zur Entwickelung kommt. Es übernimmt dann, wenn der Acardius nicht etwa vorher ganz abstirbt, das Herz des Mitzwillings die Circulation auch für die Area vasculosa des herzlosen Keimes und damit auch für diesen selbst mit.

2. Acardii, bei welchen die Acardie zwar eintritt, nachdem das Herz schon gebildet und in Function getreten ist, bei welchen sie aber auf Grundlage und durch besondere Umstände im Dotterkreislauf - ebenfalls durch primären oder secundären Herztod -eintritt, so dass es zu einem Allantois- (später Placenta-)Kreislauf gar nicht kommt. 
3. Acardii, bei welchen die Acardie erst eintritt während des bez. durch den Allantois- (später Placenta-)Kreislauf.

Die in den ersten beiden Entwickelungsperioden entstehenden Acardii (1 und 2) sind immer nur parasitäre Acardii, d. h. solche, welehe mit dem Mitzwilling körperlich verbunden sind. Sie sind nie solche, welche isolirt - natürlich neben einem Mitzwilling, aber doch von ihm körperlich getrennt -, mit besonderer Nabelschnur, oft sogar in besonderem Amnionsacke angetroffen werden. Alle Acardii der letzten Art, welche übrigens auch gewöhnlich allein kurzweg als Acardii bezeichnet werden, entstehen in der Zeit des schon rorhandenen Allantois- oder sogar des Placentakreislaufes, wie durch das Vorhandensein einer deutliehen Nabelschnur genügend bewiesen wird.

Entsprechend den Untersuchungen in den vorhergehenden Abschnitten I und II dieses Werkes, welche sich nur mit denjenigen eineiigen Zwillingen beschäftigen, die einen gemeinschaftlichen Placentakreislauf haben, im Uebrigen aber vollständig getreunt sind, werden auch die folgenden Untersuchungen sich nur auf diejenigen Acardii beziehen, welche während, bez. durch den Allantois(später Placenta-)Kreislauf solche geworden und als solche an dem Vorhandensein einer Nabelschur dentiich zu erkennen sind.

Die Entstehung der Acardii während der ersten beiden Entwickelungsperioden muss den Embryologen von Fach überlassen bleiben. Freilich werden meine Untersuchungen gewisses Licht auch auf die Entstehung der unter 2 rubricirten Acardii werfen, welche während und durch den Dotterkreislauf entstehen. - Siehe die Schlussabhandlung.

Nur diejenigen Fälle, bei deren Entstehung der Dotterkreislauf und der Allantoiskreislauf concurriren, werde ich vollständig mit bearbeiten. 